\title{
Diabetes e Gravidez: Aspectos Clinicos e Perinatais
}

\author{
Diabetes and Pregnancy: Clinical and Perinatal Features
}

Francisco Mauad Filho, Cleusa C. Dias, Roberto S. Meirelles

Sergio P. Cunha, Antonio Nogueira, Geraldo Duarte

\begin{abstract}
RESUM0
Durante a gestação, as pacientes que não se ajustam às alterações metabólicas próprias da gravidez e aquelas com alterações prévias no metabolismo dos carboidratos apresentam um aumento significativo da morbimortalidade perinatal. Visando contribuir para o aperfeiçoamento da conduta pré-natal na gestante diabética, os autores estudaram retrospectivamente 60 casos de gravidez associada ao diabetes, atendidos no Departamento de Ginecologia e Obstetrícia da Faculdade de Medicina de Ribeirão Preto, Universidade de São Paulo. A amostra estudada foi dividida em dois grupos: com controle pré-natal segundo o protocolo para a gestante diabética e encaminhadas ao Serviço para resolução da gravidez. No grupo com pré-natal adequado não ocorreram óbitos fetais ou neonatais e as complicações neonatais observadas estiveram associadas principalmente com a prematuridade. No grupo encaminhado para resolução e sem seguimento pré-natal adequado foram diagnosticados 3 casos de malformação fetal múltipla, 1 caso de hipoglicemia neonatal grave, 3 casos de prematuridade, 1 caso de retardo de crescimento intra-uterino, 1 caso de macrossomia fetal e ainda um caso de óbito neonatal. A comparação entre os grupos estudados demonstra claramente os beneficios do controle pré-natal adequado para a gestante diabética e expõe também a necessidade de uma maior integração entre a rede periférica regional e os centros de atenção terciária, permitindo o diagnóstico e o encaminhamento precoce de todos os casos de gestação complicada pelo diabetes.
\end{abstract}

PALAVRAS-CHAVE: Diabetes e gravidez. Pré-natal. Malformações fetais.

\section{Introdução}

Diabetes mellitus define um grupo de alterações genética e clinicamente heterogêneas que têm em comum a intolerância aos

Departamento de Ginecologia e Obstetrícia da Faculdade de Medicina de Ribeirão Preto, Universidade de São Paulo

Correspondência:

Francisco Mauad Filho

Departamento de Ginecologia e Obstetrícia - Faculdade de Medicina de Ribeirão Preto

Av. Bandeirantes, 3900 - CEP 14049-900 Ribeirão Preto - SP carboidratos ${ }^{10}$. A gestação é considerada diabetogênica porque se caracteriza pela resistência à insulina, associada ao aumento dos níveis séricos de estrogênio, prolactina, progesterona, cortisol e somatomamotrofina coriônica, visando manter constante o suprimento de glicose para o feto. Na gestação normal isto é compensado pelo aumento da secreção pancreática de insulina ${ }^{2}$. Nas pacientes com alterações no metabolismo dos carboidratos prévias à gestação e nas que não se ajustam às alterações próprias da gravidez, a elevação da glicemia materna acarreta hiperglicemia e hiperinsulinemia fetal, levando a um aumento da 
morbimortalidade perinatal ${ }^{7}$

Os avanços observados nas últimas duas décadas nas áreas de obstetrícia e pediatria e a melhoria do controle glicêmico durante a gestação levaram a uma significativa diminuição da morbimortalidade perinatal associada ao diabetes na gravide $z^{1,5}$. No entanto, complicações neonatais como membrana hialina, macrossomia, hipocalcemia, hiperbilirrubinemia, policitemia e hipomagnesemia ainda atingem 25\% dos recémnascidos de mães diabéticas e as malformações congênitas superam em quase três vezes aquelas observadas na população geral e contribuem como a principal causa de mortalidade perinatal $^{6,15}$.

Para minimizar as condições adversas, a paciente diabética deve ser orientada a planejar suas gestações, de modo que os níveis glicêmicos estejam normalizados antes mesmo da concepção ${ }^{3}$ e durante a gestação o controle pré-natal deve ser realizado por uma equipe multidisciplinar de assistência. A avaliação periódica dos resultados perinatais é necessária para o aprimoramento dos protocolos de conduta e, com este objetivo, analisamos no presente trabalho os resultados obstétricos, clínicos e neonatais observados em nosso Serviço.

\section{Pacientes e métodos}

Foram reunidos retrospectivamente 60 casos de diabetes e gravidez, atendidos no Departamento de Ginecologia e Obstetrícia da Faculdade de Medicina de Ribeirão Preto, USP, em conjunto com a disciplina de Endocrinologia do Departamento de Clínica Médica.

As pacientes foram agrupadas segundo a classificação de Diabetes Mellitus e Outras Categorias de Intolerância à Glicose do "National Diabetes Data Group² e da Organização Mundial da Saúde ${ }^{18,13}$.

O diagnóstico do diabetes gestacional foi estabelecido a partir de dois ou mais valores alterados no teste oral ${ }^{14}$ de tolerância à glicose. Tomou-se como limite de normalidade o valor de 90 $\mathrm{mg} / \mathrm{dl}$ para glicemia de jejum e $130 \mathrm{mg} / \mathrm{dl}$ para as demais glicemias horárias (10, 14, 20 e 2 horas).

A amostra estudada foi dividida em dois grupos:

Grupo A: pacientes acompanhadas durante o pré-natal em nosso Serviço

Grupo B: pacientes encaminhadas ao serviço para resolução imediata da gestação com ou sem diagnóstico prévio de diabetes, pacientes com menos de três controles durante o pré-natal no Serviço ou pacientes que iniciaram o controle pré- natal no Serviço após a $32^{\mathrm{a}}$ semana de gestação.

Os recém-nascidos foram avaliados no Departamento de Pediatria da Faculdade de Medicina de Ribeirão Preto considerando-se o índice de Apgar no $1^{\circ}$ e no $5^{\circ}$ minuto após o nascimento, a idade gestacional segundo o método de Capurro, exame físico e neurológico e evolução clínica durante a internação.

\section{Resultados}

\section{Resultados observados no Grupo A}

Este grupo foi constituído por 46 pacientes, sendo 27 procedentes de Ribeirão Preto e 19 de cidades vizinhas, classificadas como diabéticas tipo I ( 5 casos), tipo II (8 casos) e gestacionais (33 casos).

O início do controle pré-natal ocorreu entre 9 e 28 semanas de gestação, com mediana de $18 \pm 6$ semanas, nas diabéticas tipo I, entre 5 e 21 semanas, com mediana de $13 \pm 6$ semanas, nas diabéticas tipo II e entre 8 e 30 semanas, com mediana de $17 \pm 8$ semanas, nas diabéticas gestacionais.

Todas as pacientes diabéticas tipo I foram internadas durante a gestação para avaliação metabólica e o número de internações requeridas por paciente teve mediana de 3 . A mediana de permanência hospitalar foi de 8 dias em cada internação e o número total de dias de hospitalização foi de 146. As pacientes diabéticas tipo II também foram internadas para avaliação metabólica, excetuando-se uma paciente que foi controlada ambulatorialmente. O número de internações durante a gravidez nas diabéticas tipo II teve mediana de 2 por paciente, com mediana de permanência hospitalar de 7 dias. O total de dias de hospitalização foi de 167. Nas diabéticas gestacionais o número de internações teve mediana de 2 por paciente, destacando-se que em 6 casos o controle foi exclusivamente ambulatorial. Nos casos de internação, a mediana de permanência hospitalar foi de 4 dias, sendo que o número total de dias de hospitalização foi de 445 .

As complicações observadas durante a gravidez nas pacientes diabéticas tipo I foram 1 caso de ameaça de parto prematuro e 1 caso de infecção urinária. Nas diabéticas tipo II ocorreu 1 caso de polidrâmnio e nas diabéticas gestacionais diagnosticaram-se 9 casos de doença hipertensiva específica da gravidez, 1 caso de placenta prévia, 1 caso de polidrâmnio e 6 casos de infecção urinária.

A mediana dos perfis glicêmicos e sua amplitude semiquartil por ocasião da internação para controle e na alta hospitalar, nas pacientes do grupo A, se observam na Tabela 1. 
Tabela 1 - Mediana dos valores dos perfis glicêmicos e a amplitude semiquartil, por ocasião da internação e na alta hospitalar, nas pacientes do grupo A, segundo a classificação do diabetes (a=internação; b=alta)

\begin{tabular}{cccc} 
Perfis Glicêmicos & \multicolumn{3}{c}{ Classificação do Diabetes } \\
\hline & $\begin{array}{c}\text { Tipo I } \\
(\mathrm{n}=5)\end{array}$ & $\begin{array}{c}\text { Tipo II } \\
(\mathrm{n}=8)\end{array}$ & $\begin{array}{c}\text { Gestacional } \\
(\mathrm{n}=33)\end{array}$ \\
7 horas - $\mathrm{a}$ & $137 \pm 42$ & $109 \pm 22$ & $99 \pm 14$ \\
$-\mathrm{b}$ & $94 \pm 35$ & $109 \pm 17$ & $100 \pm 10$ \\
10 horas - $\mathrm{a}$ & $195 \pm 25$ & $186 \pm 28$ & $158 \pm 38$ \\
$-\mathrm{b}$ & $162 \pm 6$ & $174 \pm 21$ & $135 \pm 31$ \\
14 horas - $\mathrm{a}$ & $151 \pm 36$ & $141 \pm 25$ & $118 \pm 18$ \\
$-\mathrm{b}$ & $117 \pm 16$ & $132 \pm 6$ & $130 \pm 21$ \\
20 horas - $\mathrm{a}$ & $149 \pm 35$ & $145 \pm 30$ & $127 \pm 27$ \\
$-\mathrm{b}$ & $118 \pm 20$ & $126 \pm 15$ & $131 \pm 15$ \\
2 horas - $\mathrm{a}$ & $143 \pm 48$ & $118 \pm 22$ & $119 \pm 29$ \\
$-\mathrm{b}$ & $132 \pm 32$ & $112 \pm 12$ & $110 \pm 11$ \\
\hline
\end{tabular}

A diferença entre os valores encontrados por ocasião da internação e na alta hospitalar nas diabéticas tipo I não foi significativa, excetuando-se o horário das 2 horas no perfil glicêmico. Nas diabéticas tipo II, esta diferença foi significativa $(\mathrm{p}<0,05)$ em todos os horários do perfil glicêmico diário, com exceção dos valores obtidos às 2 horas, observando-se os mesmos resultados na análise dos valores referentes às diabéticas gestacionais.

As pacientes diabéticas tipo I requereram um aumento médio de $216 \%$ da dose habitual de insulina para o controle metabólico durante a gravidez e duas pacientes apresentaram hipoglicemia. No grupo de pacientes com diabetes tipo II, três pacientes foram controladas exclusivamente com a dieta e as restantes necessitaram insulina exógena. O controle metabólico nas diabéticas gestacionais foi alcançado com a instituição de dieta adequada em 19 casos, sendo que 14 pacientes utilizaram insulina exógena e, destas, três apresentaram episódios de hipoglicemia durante a internação.

A idade gestacional por ocasião do parto nas pacientes do grupo A esteve compreendida entre 30 e 38 semanas com mediana de $35 \pm 4$ semanas nas diabéticas tipo I, entre 29 e 40 semanas com mediana de $36 \pm 2$ semanas nas diabéticas tipo II e entre 34 e 41 semanas com mediana de $39 \pm 2$ semanas nas diabéticas gestacionais.

No grupo A ocorreram 47 nascimentos (houve uma gestação gemelar) e o índice de Apgar no $1^{\circ} \mathrm{e}$ no $5^{\circ}$ minuto nos recém-nascidos das diabéticas tipo I foi $\bar{X} 5 \pm 2$ e $9 \pm 0,5$, nos recém-nascidos das diabéticas tipo II foi $8 \pm 1,5$ e $10 \pm 0,5$ e nos casos de diabetes gestacional foi $9 \pm 1,5$ e $10 \pm 0,5$. As patologias observadas nos recém-nascidos estão descritas na Tabela 2 .
O tempo de permanência hospitalar dos recém-nascidos das diabéticas tipo I foi de $8 \pm 2$ dias, daqueles referentes às diabéticas tipo II foi de $6 \pm 4$ dias e dos referentes às diabéticas gestacionais foi de 3 dias.

\begin{tabular}{lccc}
$\begin{array}{l}\text { Tabela } 2 \text { - Relação entre as patologias observadas nos recém-nascidos do grupo A e o } \\
\text { tipo do diabetes. }\end{array}$ & \multicolumn{3}{c}{ Classificação do Diabetes } \\
\hline \multicolumn{1}{c}{ Patologias Neonatais } & $\begin{array}{c}\text { Tipo I } \\
(\mathrm{n}=5)\end{array}$ & $\begin{array}{c}\text { Tipo II } \\
(\mathrm{n}=8)\end{array}$ & $\begin{array}{c}\text { Gestacional } \\
(\mathrm{n}=33)\end{array}$ \\
& 2 & 1 & 5 \\
Prematuridade & - & - & 1 \\
Malformaçóes múltiplas & - & - & 1 \\
Anóxia neonatal & - & 1 & - \\
Membrana hialina grau I, macrossomia e & & & \\
prematuridade & - & 1 & - \\
Membrana hialina grau II e prematuridade & 1 & - & - \\
Membrana hialina grau III, prematuridade, \\
hipoglicemia assintomática e persistência & & & \\
do canal atrial & & & - \\
Persistência do canal atrial & 1 & - & 2 \\
Hipoglicemia & - & - & - \\
Hipoglicemia e prematuridade & - & 1 & - \\
Poliglobulia & 1 & - & 1 \\
Macrossomia & - & - & - \\
Macrossomia e hipoglicemia & - & - & 1 \\
Intolerância ao leite humano & - & 1 & - \\
\hline
\end{tabular}

\section{Resultados observados no Grupo B}

Este grupo foi constituído por 14 pacientes, das quais 7 foram encaminhadas para resolução imediata da gestação e 7 tiveram menos de 3 controles durante o pré-natal e/ou iniciaram o controle pré-natal após a $32^{\mathrm{a}}$ semana da gestação.

A classificação do diabetes nas pacientes do grupo B foi assim distribuída: diabetes tipo II (5 casos), diabetes gestacional (8 casos) e diabetes associado a doença pancreática crônica, insulinodependente, decorrente de etilismo crônico (1 caso).

Durante a gestação as complicações observadas nas diabéticas tipo II foram: 1 caso de doença hipertensiva da gravidez sobreposta à hipertensão arterial crônica com óbito fetal e 1 caso de malformação fetal múltipla e óbito fetal. Nas diabéticas gestacionais ocorreram 3 casos de polidrâmnio, sendo um associado a doença hipertensiva específica da gravidez e um associado a macrossomia fetal.

A idade gestacional ao parto nas pacientes do grupo B variou de 26 a 38 semanas com mediana de $32 \pm 5$ semanas nas diabéticas tipo II, de 34 a 42 semanas com mediana de $38 \pm 1$, 75 semanas nas diabéticas gestacionais, sendo que a paciente 
com diabetes associado a pancreatite teve parto com 35 semanas.

No grupo B ocorreram 14 nascimentos e nestes, 2 óbitos fetais. Os óbitos fetais, assim como um caso de óbito neonatal, ocorreram no grupo das diabéticas tipo II. O índice de Apgar no $1^{\circ}$ e $5^{\circ}$ minuto nos recém-nascidos das diabéticas tipo II foi respectivamente 1 e 2,1 e 3 , e 8 e 9 . Nas diabéticas gestacionais o índice de Apgar foi de $5 \pm 3$ no primeiro minuto e de $8,5 \pm 0,5$ no quinto minuto. No recém-nascido da paciente com diabetes associado o índice foi 9 e 10 . As intercorrências neonatais observadas estão descritas na Tabela 3 e atingiram 58\% dos recém-nascidos deste grupo.

O tempo de permanência hospitalar dos recém-nascidos do grupo $\mathrm{B}$ foi de $5 \pm 3$ dias.

Tabela 3 - Intercorrências neonatais nas pacientes do grupo B distribuídas segundo a classificação do diabetes.

\begin{tabular}{lccc} 
Intercorrências Neonatais & \multicolumn{3}{c}{ Classificação do Diabetes } \\
\hline & $\begin{array}{c}\text { Tipo II } \\
(\mathrm{n}=5)\end{array}$ & $\begin{array}{c}\text { Gestacional } \\
(\mathrm{n}=8)\end{array}$ & $\begin{array}{c}\text { Associado } \\
(\mathrm{n}=1)\end{array}$ \\
$\begin{array}{l}\text { Malformação múltipla (trissomia 18) } \\
\quad \text { e prematuridade }\end{array}$ & - & 1 & - \\
$\begin{array}{l}\text { Anóxia grave, malformação } \\
\text { múltipla, prematuridade e óbito neonatal }\end{array}$ & - & 1 & - \\
$\begin{array}{l}\text { Hipoglicemia grave, anóxia } \\
\text { moderada, poliglobulia, policitemia }\end{array}$ & 1 & - & - \\
$\quad$ e prematuridade \\
$\begin{array}{l}\text { Prematuridade e retardo do } \\
\text { crescimento }\end{array}$ \\
$\begin{array}{l}\text { Prematuridade, macrossomia } \\
\text { e hipoglicemia }\end{array}$ \\
\begin{tabular}{l} 
Poliglobulia \\
\hline
\end{tabular}
\end{tabular}

\section{Discussão}

A amostra estudada foi dividida em dois grupos, A e B, para permitir a avaliação do protocolo estabelecido para a gestante diabética, uma vez que as pacientes catalogadas no grupo B não fizeram seguimento pré-natal no Serviço ou o realizaram de forma insatisfatória, de acordo com o mesmo protocolo.

No grupo A o início do controle pré-natal ocorreu principalmente no segundo trimestre da gestação, o que dificulta o controle do diabetes e a prevenção das complicações clínico-obstétricas, especialmente nas pacientes com diabetes tipo I e II. Além disso, o início tardio do controle pré-natal compromete também a acurácia do diagnóstico do diabetes gestacional. No entanto, as complicações ocorridas durante a gestação não caracterizaram quadros graves, o que pode ser creditado em parte ao estrito controle a que foram submetidas as pacientes a partir do momento da admissão no Serviço. Entre as complicações observadas destacamos que a doença hipertensiva específica da gravidez (DHEG) ocorreu apenas nas pacientes com diabetes gestacional e foi a complicação mais freqüente neste grupo (27\% dos casos). Em estudos epidemiológicos com número expressivo de pacientes, não foi estabelecida uma associação significativa entre diabetes e DHEG, quando corrigidas outras variáveis predisponentes ${ }^{9}$. Por outro lado, alguns autores reportam uma maior incidência de DHEG na gestante diabética quando comparadas com gestantes normais, sendo que esta incidência aumenta com o grau de gravidade do diabetes ${ }^{8}$. Isto poderia ser atribuído ao aumento da excreção urinária de metabolitos do tromboxane A2, que ocorre nas gestantes diabéticas ${ }^{17}$.

A mediana dos perfis glicêmicos obtidos durante a internação para controle do diabetes ainda permaneceu acima dos valores ideais de $90 \mathrm{mg} / \mathrm{dl}$ para glicemia de jejum e superiores a $130 \mathrm{mg} / \mathrm{dl}$ para glicemia pós-prandial ${ }^{16}$, especialmente no que se refere aos valores da glicemia das 10 horas, nas pacientes diabéticas tipo I e II. Isto poderia estar relacionado ao aumento no clearance de insulina, a diminuição da sensibilidade à insulina ou ao pico noturno do hormônio do crescimento ou do cortisol $^{11}$. Nos demais horários, por ocasião da alta hospitalar, o perfil glicêmico se aproxima bastante ou mesmo corresponde aos padrões requeridos, em todas as pacientes do grupo A.

As pacientes do grupo A necessitaram de um número relativamente alto de internações e de dias de permanência hospitalar para controle metabólico, o que encarece sobremaneira o tratamento, além de representar um fator de estresse considerável para as pacientes e suas famílias, não obstante as vantagens advindas. A implementação de programas com ênfase no seguimento domiciliar ${ }^{4}$ ainda parece distante da nossa realidade social, mas devem ser considerados como alternativas valiosas dentro dos protocolos de assistência.

As patologias neonatais observadas nos recém-nascidos das pacientes do grupo A provavelmente se relacionaram com deficiência no controle glicêmico durante a gravide $z^{12}$, mas todos os neonatos tiveram boa evolução e requereram um tempo relativamente pequeno de hospitalização. Cabe destacar a ocorrência de um caso de malformação múltipla correspondente a uma paciente com diabete gestacional, cuja etiologia foi desconhecida, já que não há dados que permitam associá-lo com controle glicêmico inadequado por 
ocasião da organogênese. A este respeito vale ressaltar que a origem das malformações congênitas associadas ao diabetes não está ainda bem compreendida e que outros fatores, além do controle glicêmico, podem estar envolvidos ${ }^{11}$.

Os resultados aferidos no grupo A podem ser considerados, de forma geral, satisfatórios, devido à boa evolução das gestações e dos neonatos, o que não exclue a necessidade de aperfeiçoamento do protocolo, especialmente no que concerne ao controle glicêmico prévio à concepção, à melhoria do controle ambulatorial e domiciliar durante a gestação e à prevenção da prematuridade.

As pacientes do grupo B constituíram uma parcela importante das diabéticas atendidas no período estudado e apresentaram alta incidência de complicações, destacando-se dois casos de óbito fetal, sendo um deles associado a malformação fetal múltipla, além de três casos de polidrâmnio, um deles associado à macrossomia fetal, e 3 casos de malformações fetais múltiplas, tendo um deles resultado em óbito neonatal. A gravidade destes casos requer ações prementes na área de saúde voltadas ao esclarecimento da população quanto à importância do controle pré-natal e na orientação dos profissionais de saúde para o encaminhamento precoce da gestante diabética aos serviços de atenção terciária.

\section{SUMMARY}

The patients who do not adjust to the metabolic changes of pregnancy and those with previous alterations in carbohydrate metabolism show a significant increase in perinatal morbidity and mortality. In order to contribute to a better prenatal management of diabetic patients, the authors reviewed 60 cases of diabetes during pregnancy, assisted at the Department of Obstetrics and Gynecology, Faculty of Medicine of Ribeirão Preto, University of São Paulo. The sample was divided into two groups: one with prenatal care according to the Department protocol, and referred to this center for pregnancy resolution, and the other without appropriate prenatal care. In the group with prenatal care according to the Department protocol the complications observed were related to prematurity. The group without appropriate care showed 3 cases of congenital malformations, 3 cases of prematurity, 1 case of severe neonatal hypoglycemia, 1 case of macrossomia, 1 case of intrauterine growth retardation and 1 neonatal death. Comparing the groups, it became clear that the appropriate prenatal care is essential for the diabetic pregnant patient, but also that a reference center, such as this Obstetrical and Gynecological Department, must be fully integrated with the regional health centers, in order to offer assistance before and during gestation to the diabetic patients.

KEY WORDS: Diabetes and pregnancy. Prenatal care. Perinatal complications.

\section{Referências biblioğráficas}

1. Baird JD. Pregnancy and diabetes. In: The Diabetes Annual/6. Albert KGM, Krall M. Editors Elsevier Science Publishers, Amsterdam 1991:219-54.

2. Barss VA. Diabetes e gravidez. Clínicas Médicas da América do Norte 1989; 3: 753-69.

3. Bertini AM, Camano L. Diabetes melito e gravidez: Um desafio para o futuro. Femina 1996; 24: 311-24.

4. Bourgeois FJ, Duffre J. Outpatient obstetric management of women with type I diabetes. Am J Obstet Gynecol 1990, 163: 1065-73.

5. Calderon IMP, Rudge MVC. Insulinoterapia no ciclo grávido-puerperal. Benefícios e controvérsias. Femina 1997; 25:533-37.

6. Damm P, Møsted-Pedersen L. Significant decrease in congenital malformations in newborn infants of an unselected population of diabetic women. Am J Obstet Gynecol 1989; 161:1163-7.

7. Gabbe SG. Diabetes mellitus in pregnancy: Have all the problems been solved? A J M 1981; 70: 613-17.

8. Garner PR, D'Alton ME, Dudley DK, Huard P, Hardie M. Preeclampsia in diabetic pregnancies. Am J Obstet Gynecol 1990; 163: 505-8.

9. Guzick DS, Klein JR, Tyson JE, Lasky RE, Gant NF, Rosenfeld CR. Risk factors for the occurrence of pregnancy-induced hypertension. In: Zuspan FP, Symonds, EM editors. Clinical and Experimental Hypertension. Part B. Hypertension in Pregnancy. New York: Marcel Dekker, 1987 B6 (2) p. 281-97.

10. Hollingsworth DR, Moore TR. Endocrine disorders of pregnancy. In: Creasy RK, Resnick R. editors. Maternal Fetal Medicine. Principles and Practice. Philadelphia: W.B Saunders Company 1989. p. 925-88.

11. Hollingsworth DR. Pregnancy, Diabetes and Birth. A Management Guide. $2^{\text {nd }}$ ed Baltimore: Williams \& Wilkins; 1992.p. 79-95.

12. Maresh M, Beard RW, Bray CS, Elkeles RS, Wadsworth J. Factors predisposing to and outcome of gestational diabetes. Obstet Gynecol 1989; 74: 342-6.

13. National Diabetes Data Group. Classification and diagnosis of Diabetes Mellitus and other categories of glucose intolerance. Diabetes 1979; 28:1039-57.

14. O'Sullivan JR, Mahan CM.Criteria for the oral glucose tolerance test in pregnancy. Diabetes 1964, 13:278-85. 
15. Rosenn B, Miodovnick M, Combs CA, Khoury J, Siddiqi T. A. Pre-conception management of insulin-dependent diabetes: improvement of pregnancy outcome. Obstet Gynecol 1991; 77: 846-9.

16. Rudge MVC, Lima GR, Melo E, De Luca LA. Diagnóstico do diabete na gestação. II-Perfil glicêmico. Rev Bras Ginec Obstet 1988; 10: 47-51.
17. Van Assche FA, Spitz B, Hanssens M, VanGeet C, Arnout J, Vermylen J. Increased thromboxane formation in diabetic pregnancy as a possible contribuctor to preeclampsia. Am J Obstet Gynecol 1993; 168: 84-7.

18. World Health Organization Technical Report. Diabetes Mellitus Report of a WHO Study Group. Series 727, 1985. 